\title{
Implementation of monitoring the efficiency of enterprises using a special subsystem of the automated control system
}

\author{
E.V. Tuev ${ }^{1, *}$, M.F. Kozlova ${ }^{1}$, O.I. Olshevskaya ${ }^{2}$ \\ ${ }^{1}$ Reshetnev Siberian State University of Science and Technology, Krasnoyarsk, Russian \\ Federation \\ ${ }^{2}$ Siberian Federal University, Krasnoyarsk, Russian Federation \\ *E-mail: tuev_e@mail.ru
}

\begin{abstract}
The article discusses the implementation of monitoring the efficiency of enterprises using a special subsystem of the automated control system. It is shown that the analysis within the framework of the performance monitoring subsystem in the automated control system can be carried out with the integration of all available types of measurement information, for example, telemetry, command-software, etc. A list of tasks for monitoring the functioning and operability of the monitoring object, as well as predicting the behavior of the controlled object is presented. The unified structure of the enterprise with the parameters of the functioning of its objects is highlighted, which is presented using the mathematical apparatus of the vector representation of the essence of objects. The steps of the algorithm of the performance monitoring subsystem in the automated control system are developed and the features of the application of the developed monitoring subsystem in processing enterprises are presented on the example of processing bauxite, oil and solid household waste.
\end{abstract}

Keywords: monitoring, efficiency, automated control system, structure, enterprise 


\title{
Реализация мониторинга эффективности предприятий с помощью специальной подсистемы АСУП
}

\author{
Е. В. Туев ${ }^{1, *}$, М.Ф. Козлова ${ }^{1}$, О. И. Ольшевская ${ }^{2}$ \\ ${ }^{1}$ Сибирский государственный университет науки и технологий имени академика М. Ф. \\ Решетнева, Российская Федерация, г. Красноярск \\ ${ }^{2}$ Сибирский федеральный университет, Российская Федерация, г. Красноярск \\ *E-mail: tuev_e@mail.ru
}

\begin{abstract}
Аннотация. В статье рассматривается реализация мониторинга эффективности предприятий с помощью специальной подсистемы АСУП. Показано, что анализ в рамках подсистемы мониторинга эффективности в АСУП может проводиться при интеграции всех имеющихся видов измерительной информации, например, телеметрической, командно-программной и т.д. Представлен перечень задач контроля функционирования и работоспособности объекта мониторинга, а также прогнозирования поведения объекта управления. Выделена унифицированная структура предприятия с параметрами функционирования его объектов, которая представлена с помощью математического аппарата векторного представления сущности объектов. Разработаны шаги алгоритма работы подсистемы мониторинга эффективности в АСУП и представлены особенности применения разработанной подсистемы мониторинга в перерабатывающих предприятиях на примере переработки бокситов, нефти и твердых бытовых отходов.
\end{abstract}

Ключевые слова: мониторинг, эффективность, АСУП, структура, предприятие

\section{1. Введение}

В целях анализа, получаемого в рамках мониторинга предприятий в автоматизированных системах управления предприятиями (АСУП), осуществляется отработка методологических подходов и методики обработки входов системы и баз данных, составляются макеты выходных аналитических таблиц, схем и графиков. Разрабатываются алгоритмы расчета каждого показателя выходных аналитических таблиц и определяются основные направления анализа изменений структурных состояний предприятия [1, 2]. Выполняется диагностический анализ. Диагностический анализ - это комплекс исследований, проводимых с целью выявления общих тенденций развития производства и управления, разработки требований и мероприятий по улучшению системы управления предприятием. В результате диагностического анализа формируются следующие документы:

- описание предприятия и его структурной схемы;

- таблица функций предприятия; 
- характеристика задач предприятия;

- описание функций подразделений и их структурных схем;

- описание информационных потоков подразделений.

Анализ в рамках подсистемы мониторинга эффективности (ПМЭ) в АСУП может проводиться при интеграции всех имеющихся видов измерительной информации (телеметрической, командно-программной и других) и решать следующий перечень задач [68]:

- контроль функционирования объекта управления при нахождении его как в штатных, так и нештатных ситуациях;

- контроль работоспособности объекта управления при возникновении неисправностей, их диагностирование с указанием места и вида возникшей неисправности;

- прогнозирование поведения объекта управления и при наличии соответствующих исходных данных - предсказание развития как штатных, так и нештатных (аварийных) ситуаций с целью их предупреждения и недопущения.

\section{2. Анализ работы предприятий в подсистеме мониторинга}

Выделить во временном промежутке и унифицировать структуру предприятия с параметрами функционирования его объектов представляется возможным с помощью использования математического аппарата векторного представления сущности объектов [2-4]. Поэтому опишем вектор структурного состояния (BCC), представляющий собой набор объектов производства.

$$
S=\left\{W_{1}, W_{2}, \ldots, W_{\delta}, \ldots, W_{\Psi}\right\},
$$

где $S$ - вектор объектов, учитываемых в АСУП; $\delta=\overline{1, \Psi}$ - индекс, определяющий объект на предприятии; $W_{\delta}$ - вектор состояния объекта, входящего в предприятие.

Рассмотрим подробнее вектор, характеризующий объект $W_{\delta}$ :

$$
W_{\delta}=\left\{C_{1}, C_{2}, \ldots, C_{b}, \ldots, C_{B}\right\}
$$

где $C_{1}, C_{2}, \ldots, C_{B}$ - определяют параметры состояния объекта, учитываемого в подсистеме мониторинга эффективности АСУП; $b=\overline{1, B}$ - определяет индекс параметра объекта $W_{\delta}$.

Параметр $C_{b}$ модели состояний объектов предприятия дает определенную характеристику объекта $\delta$. Вектор состояний $W_{\delta}$ может содержать следующие характеристики: 
$C_{1}$ - работоспособность объекта производственной системы (например, принимать значения 1- активное состояние, 2- заменен на резервный, 0- выведен из строя);

$C_{2}$ - нагрузка в процентах от максимальной мощности на объект предприятия;

$C_{3}$ - причина вывода из строя (например, 1- по технической неисправности, 2- для временного ремонта, 3- для капитального ремонта, 0 - параметр не значим);

$C_{4}$ - количество персонала, занятого в обслуживании данного объекта;

Таким образом, вектор $W_{\delta}$ содержит элементы дискретно-числового типа, определяя профильное состояние и численные значения параметров объекта. Поэтому значимость изменения профиля объекта (вектора $W_{\delta}$ ) для оценки влияния на эффективность будет зависеть при дискретном свойстве $C_{b}$. А при числовом свойстве $C_{b}$ значимость к тому же определяется и уровнем изменения $C_{b}$ в определенные пределах, задаваемых исследователями в режиме настройки подсистемы.

С учетом (1) и (2) предлагаются следующие шаги алгоритма работы ПМЭ в АСУП.

1. Подсистема мониторинга получает параметры для расчета эффективности предприятий со всех АСУП, входящих в корпоративную сеть.

2. Подсистема мониторинга собирает информацию о текущем структурном состоянии всех предприятий.

3. Определяется текущее значение эффективности для всех производств анализируемого набора.

4. Через промежуток времени Тизм процедура определения эффективности повторяется.

5. Если анализируемые предприятия в данном наборе принимаются эффективными, то переходим к п.4.

6. Если предприятие в данной выборке не эффективно и нуждается в рекомендациях, подсистема мониторинга считывает дополнительные параметры о состоянии объектов своего предприятия (вектор S).

7. В подсистеме мониторинга анализируются:

- значения весов, полученных при определении эффективности с помощью методики оценки эффективности (например, DEA или DEA-MEXIN [5]);

- входные и выходные значения по методике DEA-MEXIN других предприятий;

- характеристики структурного состояния объектов текущего предприятия.

8. По механизму выдачи рекомендаций определяется источник изменения (снижения) эффективности и выдаются рекомендации по повышению эффективности.

9. Осуществляется переход к п.4.

\section{3. Выдача рекомендаций и корректировка с целью повышения эффективности работы предприятий}

После сбора и анализа данных, подсистема мониторинга позволяет не только предоставить руководящим субъектам информацию о работе предприятия, но и выработать 
ряд рекомендаций по повышению эффективности в ситуации, связанной с изменением эффективности.

Выделим причины изменения показателя эффективности, которыми могут быть:

- Изменения в структуре предприятия, определяются по вектору структурного состояния $S$ подсистемы мониторинга.

- Интеграция в набор анализируемых производств, нового объекта с лучшими, худшими характеристиками при использовании ПМЭ АСУП в динамическом режиме, когда в процессе работы в анализ могут быть добавлены предприятия из данной отрасли.

- Изменения во входных (выходных) характеристиках (по модели DEA), в том числе по причинам п.1 для других производств.

Все многообразие факторов роста эффективности можно классифицировать [6] по двум признакам:

- по источникам повышения эффективности, основными из которых является: снижение трудо-, материало-, фондо- и капиталоемкости производства продукции, рациональное использование природных ресурсов, экономия времени и повышение качества продукции;

- по основным направлениям развития и совершенствования производства, к которым относятся: ускорение научно-технического прогресса, повышение технико-экономического уровня производства; совершенствование структуры, внедрение организационных систем управления; совершенствование форм и методов организации производства, планирования, мотивации трудовой деятельности и другие.

Таким образом, сигналом для анализа структурного состояния текущего производства служит определение показателя эффективности по методике DEA-MEXIN [5]. Когда снижение эффективности приводит к задействованию анализа ВСС неэффективного производства.

Опишем множество ситуаций, изменений вектора структурного состояния и рекомендаций по каждой ситуации в ПМЭ АСУП.

$$
\begin{gathered}
\gamma_{h}\left(W_{\delta}, C_{1}^{\delta}, \ldots, C_{b}^{\delta}, \theta_{k}\right) \in \mathrm{Y}_{k}^{m} ; \\
r_{k}^{i} \in R_{k}^{m} ; \\
\gamma_{h}\left(W_{\delta}, C_{1}^{\delta}, \ldots, C_{b}^{\delta}, \theta_{i}\right)=r_{k}^{i},
\end{gathered}
$$


где $\gamma^{i_{h}}$ - ситуация, генерируемая изменением вектора структурного состояния; $k$ - номер ситуации, связанной с объектом $W_{\delta}$ предприятия, на котором она произошла, с параметрами $C_{b}^{\delta}$ и уровнями их значений; $k$ - номер предприятия; $r_{k}^{i}$ - рекомендация для объекта $k$-ого предприятия.

Форма ситуации (3) показывает, что произошло изменение на объекте $W_{\delta}$ в параметре $C_{b}^{\delta}$, при низменном (текущем) значении $\theta_{k}$ для $k$ - го предприятия. Множества $Y_{k}^{m}$ и $R_{k}^{m}$ характеризуют множества возможных ситуаций и рекомендаций, соответственно. Причем всегда $R_{k}^{m}>\mathrm{Y}_{k}^{m}$. Необходимо учитывать при использовании ПМЭ, что множество $Y_{k}^{m}$ ограничено штатными ситуациями, происходящими на предприятии и фиксируемыми только при снижении эффективности. Множество $R_{k}^{m}$ формируется посредством ситуаций и с помощью специалистов (технологов, пользователей и конфигураторов АСУ технологических процессов (АСУТП) и АСУП) на этапе обучения ПМЭ, так как каждое предприятие имеет свои особенности структуры, организации и функционирования, независимо от аналогичности с другими производствами.

Таким образом, тип и особенности ситуации зависят от особенностей и объекта производственной системы, на котором произошло изменение структурного состояния. При анализе и предварительном формировании рекомендаций необходимо задавать степени значимости изменений определенного структурного состояния предприятий на показатель эффективности в ПМЭ АСУП, за счет сравнения степени изменения ВСС и степени изменения показателя эффективности предприятия при прочих равных условиях.

Рассмотрим базис выдачи рекомендаций в подсистеме мониторинга.

1. Подсистема мониторинга находится в режиме выдачи рекомендаций, это означает, что проведен ряд мероприятий по сбору, обработке, анализу данных с АСУ производства и АСУТП.

2. На основе измененного ВСС формируется набор ситуаций (3).

3. Каждая ситуация в зависимости от формирующих ею факторов соотносится своей рекомендации из множества рекомендаций (4). Набор рекомендаций формируется на этапе обучения системы, поэтому в данном режиме подсистема мониторинга предлагает общую информацию о состоянии технологического процесса и производства. После обучения образуется множество ситуаций из (5), имеющих рекомендации и множество внештатных ситуаций ${ }^{*} \gamma^{i}{ }_{h}$, имеющих более ограниченный набор рекомендаций (например, обратиться к диспетчеру и выслать ремонтно-наладочный наряд на объект). 
4. Применяя рекомендации, предпринимается ряд шагов по реконфигурации связей и объектов предприятия, для восстановления показателя эффективности. Такими шагами по рекомендациям могут быть, например.

- д довести характеристику состояния до характеристики $\mathrm{Cb}$ аналогичного объекта эффективно работающего предприятия, за счет повышения качества технического обслуживания (привлечения дополнительного персонала);

- произвести наладочный капительный ремонт, с целью устранения неисправностей и модернизации объекта;

- предложить в ближайшем будущем заменить объект на более современный и менее затратный по своим характеристикам.

5. После применения рекомендаций, в следующем периоде замерить показатель эффективности повторно, с целью оценить, насколько повысилась эффективность текущего предприятия, либо произошло снижение эффективности за счет привлечения дополнительных ресурсов, применяя рекомендации подсистемы мониторинга. В первом случае мониторинг продолжается в штатном режиме, во втором - подсистема мониторинга запрашивает повторный анализ применяемых рекомендаций с целью их корректировки и уточнения.

\section{4. Особенности применения разработанной подсистемы мониторинга в перерабатывающих предприятиях}

Применение подсистемы мониторинга эффективности в АСУП имеет ряд особенностей связанных с оценкой перерабатывающих предприятий. Важным существенным отличием перерабатывающих предприятий, на которых используется ПМЭ АСУП, является многокомпонентный состав сырья. Типами многокомпонентного сырья являются комплексные соединения, добываемые из недр Земли [7, 8]; твердые бытовые отходы, состав которых зависит от степени потребления населением разных типов продуктов и некоторых других факторов [9-11].

Еще одну особенность представляет дифференциация типа сырья по комплексному составу компонентов переработки, которые используются в производстве для выпуска продукции. Проблема дифференциации сырья возникает при оценке предприятий, находящихся в разных регионах, и соответственно использующих сырье с отличающимся типом набора компонентов переработки, а также концентрацией этих компонентов в сырье.

Рассмотрим особенности производств, связанных с применением методики DEAMEXIN, для их оценки, на примере переработки трех видов сырья:

- Переработка бокситов. 
- Переработка нефти.

- Переработка твердых бытовых отходов.

В таблицах 1-3 представлены входные и выходные параметры при оценке эффективности соответствующих предприятий.

\section{1. Переработка бокситов}

Бокситы являются главным сырьем для производства глинозема - продукта для получения алюминия. Бокситы состоят из гидроксидов алюминия, оксидов железа и кремния, содержание глинозёма в промышленных бокситах колеблется от 40 \% до 60 \% и выше. В результате переработки боксита на глинозем получают побочный продукт - красный шлам, который отводится в отвал. Для хранения откачиваются миллионы тонн шламов ежегодно. Красные шламы являются техногенными отходами и несут серьезную экологическую угрозу. При переработке высокожелезистых бокситов существует возможность комплексного использования сырья [7]. В зависимости от природы породообразующего минерала выделяются 3 группы бокситов: а) моногидратные, содержащие глинозём в одноводной форме (диаспор, бемит), б) тригидратные, содержащие глинозём в трёхводной форме (гиббсит) и в) смешанные, в которых сочетаются обе формы.

Содержание оксида железа, а также других веществ влияет на возможности выработки алюминиевого глинозема, а также на отходы производства. Продукты переработки представлены в таблице 1.

Таблица 1. Продукты переработки бокситов.

\begin{tabular}{ll}
\hline Вход & Выходы \\
\hline & глинозем \\
Бокситы & карбонатные щелочи \\
& нефелиновый шлам \\
& галлиевый раствор \\
\hline
\end{tabular}

В результате анализа по методике DEA-MEXIN получаем техническую эффективность производств по выпуску полезных компонентов исключая красный шлам [10].

\section{2. Переработка нефти}

Нефть - природная маслянистая горючая жидкость, состоящая из сложной смеси углеводородов и некоторых органических соединений. Состоит из углеводородов (алканов, циклоалканов, аренов - ароматических углеводородов - и их гибридов) и соединений, содержащих, помимо углерода и водорода, гетероатомы - кислород, серу и азот. Соединения сырой нефти - это сложные вещества, состоящие из пяти элементов - C, H, S, O и N, причем 
содержание этих элементов колеблется в пределах 82-87\% углерода, 11-15\% водорода, 0,01$6 \%$ серы, 0-2\% кислорода и 0,01-3\% азота. По химическому составу нефть также разнообразна. Поэтому говорить о среднем составе нефти или «средней» нефти можно только условно. Нефть представляет собой смесь около 1000 индивидуальных веществ, из которых большая часть - жидкие углеводороды (> 500 веществ или обычно 80-90 \% по массе) и гетероатомные органические соединения (4-5\%), преимущественно сернистые (около 250 веществ), азотистые (> 30 веществ) и кислородные (около 85 веществ), а также металлоорганические соединения (в основном ванадиевые и никелевые); остальные компоненты - растворённые углеводородные газы $\left(\mathrm{C}_{1}-\mathrm{C}_{4}\right.$, от десятых долей до 4 \%), вода (до $10 \%$ ), минеральные соли (главным образом хлориды, 0,1-4000 мг/л и более), растворы солей органических кислот и другие, механические примеси (частицы глины, песка, известняка) [8]. Продукты переработки нефти представлены в таблице 2.

Таблица 2. Продукты переработки нефти.

\begin{tabular}{ll}
\hline Вход & Выходы \\
\hline & бензин (разных сортов) \\
& керосин \\
& дизельное топливо \\
масла & гудрон \\
Сорая нефть & покс \\
парафины \\
другие вещества
\end{tabular}

Таблица 2 содержит не полный список веществ, получаемых из нефти, но отражает компонентный состав выпуска продукции из многокомпонентного сырья.

\section{3. Переработка твердых бытовых отходов}

Твердые бытовые отходы (ТБО) представляют собой продукты жизнедеятельности населения города и других поселений и включают различные материалы (картон, бумага, стекло, химические реактивы и другое) в пропорциях, зависящих от развитости региона и особенностей менталитета населения по потреблениям и предпочтениям промышленных продуктов и услуг. Наблюдается большое разнообразие компонентов, входящих в состав единицы отходов (тонны или кубического метра), но не все из них могут быть выделены и применены повторно. В связи с этим возникает определенный набор компонентов отходов, 
которые выделяются и используются с помощью применяемых технологий при данном научно-техническом прогрессе и целесообразной себестоимости их переработки. В таблице 3 представлены наиболее используемые после переработки вещества.

Таблица 3. Продукты переработки ТБО.

\begin{tabular}{ll}
\hline Вход & Выходы \\
\hline картон \\
бумага \\
пластики высокого и низкого давления \\
черные металлы \\
цветные металлы \\
стекло \\
текстиль \\
полимеры \\
дерево \\
другие вещества
\end{tabular}

Каждый объект, учитываемый в АСУП перерабатывающего предприятия твердых бытовых отходов, имеет 4 основных контролируемых параметра и ряд дополнительных (от 5 до 25, в зависимости от сложности объекта управления, а также до 40 и более при анализе объектов с тонкими настройками и высоким уровнем автоматизации). Данные для определения объектов и их параметров были взяты в [9-11], а также при посещении российских предприятий, перерабатывающих ТБО. Объекты, анализируемые в АСУП предприятия перерабатывающего твердые бытовые отходы представлены в таблице 4.

Таблица 4. Объекты и их параметры, анализируемые в АСУП перерабатывающих предприятий.

\begin{tabular}{llcc}
\hline Номер п/п & \multicolumn{1}{c}{ Объект для учета в АСУП } & \multicolumn{2}{c}{$\begin{array}{c}\text { Количество анализируемых } \\
\text { параметров }\end{array}$} \\
\hline 1 & Подающая линия сырья & 4 \\
2 & Линия сортировки & 25 \\
& Линия выделения мелких & фракций & \multicolumn{1}{c}{15} \\
3 & удобрений & 5 \\
4 & Линия отделения отходов (хвостов) & 33
\end{tabular}


Modern Innovations, Systems and Technologies, 2021, 1(2)

\begin{tabular}{lll}
\hline 6 & Пресс пластика, бумаги, картона & 35 \\
7 & Пресс жести и алюминиевых банок & 38 \\
8 & Агрегат переработки пластика & 26 \\
9 & Агрегат переработки картона & 20 \\
10 & Агрегат переработки цветного металла & 35 \\
11 & Агрегат переработки черного металла & 35 \\
12 & Агрегат переработки стекло & 25 \\
13 & Агрегат переработки ПНД и Пвд & 30 \\
14 & Агрегат переработки пластиковых бутылок & 29 \\
15 & Линия подачи на весы & 5 \\
16 & Учетные автоматизированные весы & 15 \\
\hline
\end{tabular}

\section{5. Заключение}

Рассмотренные особенности методики на примерах переработки бокситов, нефти и ТБО показывают широту применения разработанной ПМЭ АСУП. Реализация предложенной методики мониторинга осуществлялась на предприятиях, перерабатывающих твердые бытовые отходы, поэтому авторами приведены особенности их функционирования и структуры, отмеченные ранее в публикациях [9-15]. Апробация ПМЭ АСУП выполнена на объектах одной из рассмотренных областей применения методики оценки эффективности, а именно, перерабатывающих производств.

\section{Список литературы}

[1] Federico, G.S., Uncertain optimal inventory as a strategy for enterprise global positioning / G.S. Federico, F.R. Beatriz, A.M. Gil-Lafuente, F. Juan // Lecture Notes in Economics and Mathematical Systems. - 2015. - № 675. - P. 29-42.

[2] Охтелев, М.Ю. Интеллектуальные технологии мониторинга и управления структурной динамикой сложных технических объектов / М.Ю. Охтелев, Б.В. Соколов, Р.М. Юсупов // М.: Наука, 2006. - 410 с.

[3] Лычев, А.В. Развитие и обобщение моделей методологии анализа среды функционирования для анализа деятельности сложных систем: Автореф. дис. канд. физ.-мат. наук. / Лычев А.В. - М., 2008. $-26 \mathrm{c}$.

[4] Налимов, В.В. Теория эксперимента / В.В. Налимов. - М.: Наука, 1971. - 207 с.

[5] Kovalev, I. The Efficiency Analysis of Automated Lines of Companies Based on DEA Method / I. Kovalev, P. Zelenkov, S. Ognerubov // Lecture Notes in Economics and Mathematical Systems. - 2015. - № 675. - P. 107-115. 
[6] Фролова, Т.А. Экономика предприятия: конспект лекций / Т.А. Фролова. - Таганрог: ТРТУ, 2005.

[7] Иванов, А.И. Комплексная переработка бокситов / А.И. Иванов, Г.Н. Кожевников, Ф.Г. Ситдиков, Л.П. Иванова. - Екатеринбург: РИО УрОРАН, 2003. - 182 с.

[8] Леффлер, У.Л. Переработка нефти. - 2-е изд. пересмотренное. / Пер. с анг. - М.: Олимпбизнес, 2004. -224 c.

[9] Новожилов, А.А. Метод DEA для анализа функционирования предприятий по переработке твердых бытовых отходов /А.А. Новожилов // Информатика и системы управления. - 2010. - №1(23). - C.98-103.

[10] Новожилов, А.А. Необходимость глубокой переработки отходов в условиях Красноярского края /А.А. Новожилов // Вестник НИИ СУВПТ: сб. научн. трудов / под общей ред. профессора Н.В. Василенко. - Красноярск: НИИ СУВПТ, 2007. - № 25. - С.27-33.

[11] Zenyutkin, N.V. DEA method application to evaluating the efficiency of heat and wind generating complexes / N.V. Zenyutkin, I.V. Kovalev, M.V. Karaseva, V.V. Dvirny, A.A. Melkomukov, N.V. Lukonin // IOP Conf. Series: Materials Science and Engineering. - 2020. - № 734. - P. 012213. doi:10.1088/1757-899X/734/1/012213.

[12] Новожилов, А.А. Аналитическая система по организации переработки отходов / А.А. Новожилов // Вестник НИИ СУВПТ: сб. научн. трудов / под общей ред. профессора Н.В. Василенко. Красноярск: НИИ СУВПТ, 2007. - № 25. - С.137-144.

[13] Kovalev, I.V. Combined efficiency margin in the implementation of the DEA method / I.V. Kovalev, A.S. Kuznetsov, N.V. Zenutkin, E.A. Morozov, A.V. Zeitler // IOP Conf. Series: Materials Science and Engineering. - 2020. - № 734. - P. 012036. doi:10.1088/1757-899X/734/1/012036.

[14] Новожилов, А.А. Моделирование перерабатывающей отрасли /А.А. Новожилов// Молодежь и наука: начало XXI века. сб. мат. V Всероссийской научно-технической конференции студентов, аспирантов и молодых ученых. - Красноярск: СФУ, 2009. - 336-338 с.

[15] Kovalev, I.V. Monitoring of production processes in the waste processing industry and their impact on the environment / I.V. Kovalev, D.I. Kovalev, A.A. Voroshilova, E.V. Tuev, E.V. Tueva // IOP Conf. Series: Earth and Environmental Science. - 2021. - № 677. - P. 052115. doi:10.1088/17551315/677/5/052115. 